Published in final edited form as:

Biochemistry. 2006 March 21; 45(11): 3548-3553.

\title{
Absence of Substrate Channeling between Active Sites in the Agrobacterium tumefaciens IspDF and IspE Enzymes of the Methyl Erythritol Phosphate Pathway'
}

\author{
Christian Lherbet ${ }^{\dagger}$, Florence Pojer§, Stéphane B. Richard§, Joseph P. Noel§, and C. D. \\ Poulter ${ }^{\dagger}$ \\ $\dagger$ Department of Chemistry, University of Utah, Salt Lake City, Utah 84112
}

§Jack Skirball Chemical Biology and Proteomics Laboratory, The Salk Institute for Biological Studies, La Jolla, CA 92037

\footnotetext{
${ }^{\dagger}$ Supported by NIH Grants GM25527 (CDP) and AI51438 (JPN)

poulter@chem.utah.edu, Phone: 801-581-6685; FAX: 801-581-4391.

${ }^{1}$ Abbreviations:

Amp

ampicillin

ATP

adenosine 5'-triphosphate

CTP

cytosine $5^{\prime}$-triphosphate

CDP-ME

4-diphosphocytidyl-2- $C$-methyl-D-erythritol

CDP-MEP

4-diphosphocytidyl-2-C-methyl-D-erythritol 2-phosphate

cMEDP

2-C-methyl-D-erythritol 2,4-cyclodiphosphate

DMAPP

dimethylallyl diphosphate

HDMAPP

4-hydroxydimethylallyl diphosphate

IPP

isopentenyl diphosphate

IPTG

isopropyl 1-thio-(-D-galactopyranoside

MEP

$2 C$-methyl-D-erythritol 4-phosphate

MVA

mevalonate

PMSF

phenylmethylsulfonyl fluoride
}

SDS-PAGE 


\section{Abstract}

The conversion of $2 C$-methyl-D-erythritol 4-phosphate (MEP) to $2 C$-methyl-D-erythritol 2,4cyclodiphosphate (cMEDP) in the MEP entry into the isoprenoid biosynthetic pathway occurs in three consecutive steps catalyzed by the IspD, IspE, and IspF enzymes, respectively. In Agrobacterium tumefaciens the ispD and isp $F$ genes are fused to encode a bifunctional enzyme that catalyzes the first (synthesis of 4-diphosphocytidyl-2- $C$-methyl D-erythritol) and third (synthesis of $2 C$-methyl-D-erythritol 2,4-cyclodiphosphate) steps. Sedimentation velocity experiments indicate that the bifunctional IspDF enzyme and the IspE protein associate in solution raising the possibility of substrate channeling among the active sites in these two proteins. Kinetic evidence for substrate channeling was sought by measuring the time courses for product formation during incubations of MEP, CTP, and ATP with the IspDF and IspE proteins with and without an excess of the inactive IspE (D152A) mutant in presence or absence of 30\% (v/v) glycerol. The time dependencies indicate that the enzyme-generated intermediates are not transferred from the IspD active site in IspDF to the active site of IspE or from the active site in IspE to the active site in the IspF module of IspDF.

\section{Keywords}

bifunctional; IspDF; IspE; non-channeling

Isopentenyl diphosphate (IPP) and dimethylallyl diphosphate (DMAPP) are the two fivecarbon building blocks used in nature to construct the hydrocarbon skeletons of isoprenoid compounds ${ }^{1}$. IPP and DMAPP are synthesized by two different biosynthetic pathways - the mevalonate (MVA) pathway ${ }^{2}$ found in mammals, plants, fungi and gram-positive bacteria and the recently discovered $2 C$-methyl-D-erythritol 4-phosphate (MEP) pathway found in algae, cyanobacteria, eubacteria and plant chloroplasts ${ }^{3}$.

The MEP biosynthetic pathway ${ }^{4}$ is outlined in Scheme 1 . Three central steps are required to convert MEP into 2-C-methyl-D-erythritol 2,4-cyclodiphosphate (cMEDP), a methylerythritol derivative containing a unique cyclic diphosphate moiety. Methylerythritol 4-phosphate cytidylyltransferase, encoded by ispD, condenses MEP with cytosine 5 '-triphosphate (CTP) to give 4-diphosphocytidyl-2-C-methyl $\mathrm{D}$-erythritol (CDP-ME) and inorganic pyrophosphate $\left(\mathrm{PP}_{\mathrm{i}}\right)^{5}$. CDP-ME kinase encoded by ispE catalyzes an ATP-dependent phosphorylation of CDP-ME to produce 4-diphosphocytidyl-2C-methyl-D-erythritol 2-phosphate (CDP-MEP) ${ }^{6}$, 7 . Finally, CDP-MEP is converted to cMEDP with concomitant release of CMP by methylerythritol 2,4-cyclodiphosphate synthase, encoded by isp $F^{8}$. In most organisms, ispD and $i s p F$ are encoded by separate genes. However, bioinformatic analysis of deposited genomic data revealed 14 different instances in which ispD and ispF are fused genes resulting in the production of a single bi-functional polypeptide possessing both IspD and IspF activity 9,10 , 11. Biochemical analysis confirms that the bifunctional enzyme catalyzes the first and third steps in the conversion of MEP to cMEDP.

This unusual arrangement of enzymes within a metabolic pathway could potentially enhance flux through the MEP pathway by direct metabolite channeling in a putative IspDF/IspE complex. Although no evidence for protein-protein interactions among the individual IspD, IspE and IspF proteins from $E$. coli has been presented, the IspDF fusion protein and the IspE protein from $C$. jejuni and A. tumefaciens are reported to associate ${ }^{11}$. We now report kinetic studies that address the issue of metabolite channeling in the A. tumefaciens enzymes. 


\section{Experimental Procedures}

\section{Materials}

Restriction enzymes were purchased from New England Biolabs. KlenTaq-LA DNA Polymerase Mix was purchased from Sigma. Ni-NTA resin was from Qiagen. MEP was obtained from Echelon Biosciences, Inc. IPTG were from USB. ATP, CTP were obtained from Sigma. $\gamma-\left[{ }^{32} \mathrm{P}\right]$ ATP and $\alpha-\left[{ }^{32} \mathrm{P}\right]$ CTP was from NEN. All other chemicals were purchased from Sigma-Aldrich. TLC plates (Polygram Sil N-HR) were purchased from Macherey \& Nagel. ImageQuant 5.2 (Molecular Dynamics) was used to quantify the radioactivity on TLC plates.

\section{Cloning}

PCR amplification of Agrobacterium tumefaciens IspDF from genomic DNA (ATCC33970D) was performed with primers CL0610DFC58sen and CL0610DFC58ant and Klen Taq polymerase according to the following procedure: $94^{\circ} \mathrm{C}(3 \mathrm{~min}), 61^{\circ} \mathrm{C}(2 \mathrm{~min})$ for $1 \mathrm{cycle}$, following by $68^{\circ} \mathrm{C}(2 \mathrm{~min}), 94^{\circ} \mathrm{C}(45 \mathrm{~s}), 61^{\circ} \mathrm{C}(45 \mathrm{~s})$, for 30 cycles (Table 1$)$. The PCR product was purified using the GFX Gel Band Purification Kit and ligated into pGEM-Teasy with T4 ligase (Promega). The resulting plasmid was transformed into JM109 cells, and positive colonies were detected by blue/white screening in presence of IPTG and X-Gal. Constructs possessing the correct insert were chosen based upon restriction digests with NdeI and BamHI and were confirmed by automated DNA sequencing (University of Utah Core Facility). The NdeI/BamH1 insert was subcloned into the NdeI and BamHI sites of pET-15b resulting in an expression clone containing an $\mathrm{N}$-terminal $\mathrm{His}_{6}$-tag sequence. The desired construct, pET15b/AtDF, was used to transform electrocompetent XL1-blue cells. Positive constructs based upon restriction enzyme analysis with $\mathrm{NdeI}$ and BamHI were sequenced.

IspE was obtained by PCR amplification using primers CL0429EC58sen and CL0429EC58ant and Agrobacterium tumefaciens genomic DNA (ATCC33970D) using the following PCR settings: $94^{\circ} \mathrm{C}(2 \mathrm{~min}), 61^{\circ} \mathrm{C}(2 \mathrm{~min})$ for $1 \mathrm{cycle}$, following by $68^{\circ} \mathrm{C}(2 \mathrm{~min}), 94^{\circ} \mathrm{C}(30 \mathrm{~s}), 61^{\circ}$ $\mathrm{C}(30 \mathrm{~s})$, for 30 cycles. The IspE expression clone was obtained using a procedure like that described above for IspDF.

The IspE(D152A) site directed mutant was obtained using the Quick Change kit from Stratagene. The mutated gene was amplified using primers AMAISPE007 and GCAMAISPE007 (Table 1) to give pET15b/AtE(D158A), which was then sequenced to verify the mutation.

\section{Expression of IspDF, IspE and IspE(D152A) and Protein Purification}

BL21(DE3)pLysS was transformed with $\mathrm{pET} 15 \mathrm{~b} / \mathrm{At} \mathrm{DF}, \mathrm{pET} 15 \mathrm{~b} / \mathrm{A} t \mathrm{E}$ and $\mathrm{pET} 15 \mathrm{~b} / \mathrm{At} \mathrm{E}$ (D152A) and $5 \mathrm{~mL} \mathrm{LB}$ cultures (100 $\mu \mathrm{g} / \mathrm{mL}$ ampicillin) of the transformants were grown overnight at $37^{\circ} \mathrm{C}$, with shaking. Four cultures (two for each enzyme), each containing $1 \mathrm{~L}$ of $\mathrm{LB}, 100 \mu \mathrm{g} / \mathrm{mL}$ ampicillin, were inoculated with $2 \mathrm{~mL}$ of the overnight culture and were grown at $37^{\circ} \mathrm{C}$, with shaking at $250 \mathrm{rpm}$, until $\mathrm{OD}_{600}=0.6$. IPTG was then added to $1 \mathrm{mM}$ (final concentration), incubation was continued for $5.5 \mathrm{~h}$, and cells were harvested by centrifugation and stored at $-80^{\circ} \mathrm{C}$.

Cell paste was resuspended in $50 \mathrm{mM}$ sodium phosphate, $\mathrm{pH}=8.0,300 \mathrm{mM} \mathrm{NaCl}, 10 \mathrm{mM}$ imidazole (lysis buffer). Complete Protease Inhibitor Cocktail Tablets (Roche) and lysozyme $(1 \mathrm{mg} / \mathrm{mL})$ were added, the cells were lysed by sonication, cellular debris was removed by centrifugation, and the His-tagged enzymes were purified by chromatography on Ni-NTA agarose (Qiagen). 
$\mathrm{CaCl}_{2}$ (2.5 mM final concentration) was added to the solutions of His 6 -tagged IspDF, IspE and $I s p E(D 152 A)$, each containing a thrombin cleavage site. Thrombin (50 units) was added to $6 \mathrm{mg}$ of each protein in elution buffer $(50 \mathrm{mM}$ sodium phosphate, $\mathrm{pH}=8.0,300 \mathrm{mM} \mathrm{NaCl}$, $250 \mathrm{mM}$ imidazole) at $4^{\circ} \mathrm{C}$. After $12 \mathrm{~h}$, thrombin was removed by adding benzamidine-agarose (Sigma). The samples were filtered and dialyzed with the lysis buffer to reduce the imidazole concentration below $10 \mathrm{mM}$. Finally, Ni-NTA resin was added, and unproteolyzed proteins and the $\mathrm{His}_{6}$ fragment were removed by Ni-NTA chromatography. The flow through fractions were concentrated with a Centriprep-10 unit (Centricon) and dialyzed against $0.1 \mathrm{mM} \mathrm{Tris} \cdot \mathrm{HCl}$ $\mathrm{pH} 7.6$ (measured at $37^{\circ} \mathrm{C}$ ) using a 10,000 molecular weight cut-off dialysis cassette (Pierce). The protein concentrations were determined by the Bradford method 12 .

\section{Analytical Ultracentrifugation}

Sedimentation velocity experiments were performed using a Beckman XL-1 analytical ultracentrifugation with absorbance optical detection. Three different concentrations of purified wild-type IspE and IspE(D152A) in $25 \mathrm{mM}$ HEPES, pH 7.5 and $100 \mathrm{mM} \mathrm{NaCl}$ were used for sedimentation studies. Protein samples were centrifuged at a rotor speed (An50 Ti) of $42,000 \mathrm{rpm}$, and absorbance data at $280 \mathrm{~nm}$ were collected. Analyses of the sedimentation velocity data were performed using the program SEDFIT ${ }^{13}$.

\section{Enzyme Generated Products}

Enzymatic reactions were carried out at $37^{\circ} \mathrm{C}$ in $0.1 \mathrm{mM}$ Tris $\cdot \mathrm{HCl}$ buffer, $\mathrm{pH}$ 7.6, containing $5 \mathrm{mM}$ DTT, $10 \mathrm{mM} \mathrm{MgCl}_{2}, 150 \mu \mathrm{M}$ CTP, $150 \mu \mathrm{M}$ ATP, $500 \mu \mathrm{M}$ racemic MEP, $0.24 \mu \mathrm{M}$ IspDF, $0.31 \mu \mathrm{M}$ IspE and varying concentrations $(0,0.3,0.6,1.4,3.1,6.3,12.5 \mu \mathrm{M})$ of IspE (D152A) in a final volume of $\left.50 \mu \mathrm{L} .{ }^{32} \mathrm{P}\right] \mathrm{NTPs}$ were diluted from $5 \mathrm{mM}$ stock solutions of $40 \mu \mathrm{Ci} / \mu \mathrm{mol} \mathrm{CTP}$ and $320 \mu \mathrm{Ci} / \mu \mathrm{mol}$ ATP. In control experiments, samples were pre-incubated for $5 \mathrm{~min}$ and $20 \mathrm{~min}$, respectively, in the presence of all substrates, except for radio-labeled ATP and CTP, or enzyme. Reactions were initiated by addition of CTP. After $15 \mathrm{~min}$, the reactions were quenched with $50 \mu \mathrm{L}$ of methanol and were put on ice. TLC analysis (Polygram Sil N-HR; Macherey \& Nagel) was performed by spotting $3.5 \mu \mathrm{L}$ of the reaction mixture and developing the plates with $n$-propanol / ethyl acetate / $\mathrm{H}_{2} \mathrm{O}(6: 1: 3$, v/v/v). Radioactivity was quantified with a Molecular Dynamics Typhoon 8600 Phosphorimager.

\section{Time Course Studies}

A solution of $500 \mu \mathrm{M}$ MEP (racemic), $0.24 \mu \mathrm{M}$ IspDF and $0.31 \mu \mathrm{M}$ IspE in $0.1 \mathrm{M}$ Tris. $\mathrm{HCl}$ buffer, $\mathrm{pH} 7.6\left(37^{\circ} \mathrm{C}\right)$, containing $5 \mathrm{mM}$ DTT, in a final volume of $150 \mu \mathrm{L}$ was pre-incubated for $10 \mathrm{~min}$ at $37^{\circ} \mathrm{C}$. ATP $(150 \mu \mathrm{M})$ and $\left.\alpha-{ }^{32} \mathrm{P}\right] \mathrm{CTP}(150 \mu \mathrm{M}, 40 \mu \mathrm{Ci} / \mu \mathrm{mol})$ were added sequentially to initiate the reaction. At various times, $6 \mu \mathrm{L}$ portions of the mixture were removed and quenched with $6 \mu \mathrm{L}$ of methanol. After $61 \mathrm{~min}$, additional $0.5 \mu \mathrm{g}$ portions of each enzyme were added to the reaction mixture. Identical reactions were run in presence of 3.1 $\mu \mathrm{M}$ IspE or a mixture of $3.1 \mu \mathrm{M}$ IspE and $31 \mu \mathrm{M}$ IspE(D152A). After $61 \mathrm{~min}, 0.5 \mu \mathrm{g}$ of IspDF and $0.5 \mu \mathrm{g}$ of IspE were added to the reaction mixtures. The reactions were repeated in $0.1 \mathrm{M}$ Tris $\cdot \mathrm{HCl}$ buffer containing $30 \%(\mathrm{v} / \mathrm{v})$ glycerol at $\mathrm{pH} 7.6\left(37^{\circ} \mathrm{C}\right)$ with $3.12 \mu \mathrm{M}$ IspE or 3.12 $\mu \mathrm{M}$ IspE and $31 \mu \mathrm{M}$ IspE(D152A). After $61 \mathrm{~min}, 0.5 \mu \mathrm{g}$ of IspDF and $0.5 \mu \mathrm{g}$ of IspE were added to the reaction mixtures.

\section{Results}

\section{Expression of IspDF, IspE, and IspE(D152A) and Protein Purification}

A. tumefaciens IspDF and IspE were amplified by PCR and cloned into pET15b. Expression of the genes in E. coli BL21(DE3) cells provided $\mathrm{His}_{6}$-tagged proteins that were easily purified by chelation chromatography on Ni-NTA resin resulting in protein judged to be $>95 \%$ pure 
based upon SDS-PAGE analysis. The His 6 -tags were removed with thrombin digestion and re-purification over benzamidine sepharose and addition Ni-NTA resin.

An inactive mutant of A. tumefaciens IspE was obtained by replacing the catalytically essential Asp152 with Ala. D152 is located in a conserved LGADVP motif found in the $A$.

tumefaciens and E. coli proteins. Analysis of the X-ray structure of $E$. coli IspE suggests that the aspartate acts as a general base to deprotonate the tertiary hydroxyl group of CDP-ME during its phosphorylation by $\mathrm{ATP}^{14}$. The mutant gene was cloned into pET15b and the His $_{6}$-tagged protein was purified as described above. The $\mathrm{N}$-terminal His ${ }_{6}$-tag preceding a thrombin protease cleavage site was removed and the non-tagged protein was used as is in subsequent experiments.

\section{Analytical Ultracentrifugation of IspE and IspE(D152A)}

Sedimentation velocity experiments were carried out to accurately measure the oligomerization state of mutant and wild-type IspE. The sedimentation coefficient $(s)$ of both IspE proteins at different concentrations is identical $(\sim 2.1 s)$ and is consistent with a monomeric state for this small molecule kinase assuming a globular conformation. The sedimentation experiments indicated that wild-type IspE and the D152A mutant exist as monomers (99\%) in equilibrium with $\sim 1 \%$ of an undetermined form (Figure 1). These observations are consistent with data in the literature ${ }^{11}$. These results demonstrate that the D152A mutation does not substantially alter the overall conformation of the protein relative to wild-type IspE.

\section{Effect of IspE(D152A) Mutant on the Conversion of MEP to cMEDP by wild type enzymes}

First, IspE(D152A) was checked for activity by incubating the purified enzyme with MEP, CTP, ATP and wild-type IspDF under the standard conditions for product formation and analysis. Formation of cMEDP was not detected at IspE(D152A) concentrations up to 300 $\mu \mathrm{M}$ (data not shown). Assuming a detection limit of at least $20 \%$ for cMEDP in the product mixture, we estimate that the D152A mutant is at least 10,000 times less active than wild-type IspE. This loss of activity is consistent with the proposal that D152 acts as a general base required for deprotonation and subsequent phosphorylation of the tertiary hydroxyl group of CDP-ME ${ }^{14}$.

Radiolabeled ATP and CTP were used to monitor the formation of CDP-ME, CDP-MEP, CMP, and cMEDP. As illustrated in Figure 2, addition of IspE(D152A) during the 15 minute preincubation of a mixture of MEP, $\left[\alpha-{ }^{32} \mathrm{P}\right] \mathrm{CTP}$, and $\left[\gamma^{-32} \mathrm{P}\right]-\mathrm{ATP}$, IspDF, and wild-type IspE did not alter the product distribution at IspE(D152A) / IspE ratios $>40: 1$. Varying the preincubation time prior to initiating the assay did not alter the distribution of products.

\section{Time Course for Formation of Products in the Presence and Absence of IspE(D152A)}

Following the protocol described above, the time course for conversion of MEP to cMEDP using IspDF and IspE was monitored by TLC (Figure 3). The intermediates and final product, CDP-MEP, CDP-ME, CMP and cMEDP, were resolved and quantified by phosphorimaging 9 . Plots of the concentrations of the intermediates and the final product cMEDP are shown in Figure 4. For the experiment illustrated in Figure 4A, the concentrations of IspDF and IspE were chosen so that MEP was rapidly converted to CDP-ME, followed by a slower conversion of CDP-ME to CDP-MEP. Radioactivity from CMP, or a degradation product that accumulated with time, tailed into the spot for CDP-ME, which interfered with our ability to accurately measure the CDP-ME concentration after $\sim 10$ minutes. The concentration of CDP-MEP reached a maximum after $\sim 15$ minutes and never accumulated to levels above $15 \%$ of the total. The concentration of IspE was increased 10-fold for the time course shown in Figure 4B. In this case, the concentration of CDP-MEP reached a maximum of $\sim 30 \mu \mathrm{M}$ (corresponding to approximately $20 \%$ of the total) within 5 minutes and slowly 
decreased as the reactions proceeded. These results indicate that the relative catalytic efficiency of the IspF subunit is high and that the limiting step is the formation of CDP-MEP even when the IspE protein in present in a 13-fold molar excess. Figure $4 \mathrm{C}$ shows a time course for the same substrate and enzymes concentrations used in Figure 4B, except for the addition of a 10fold molar excess of the inactive IspE(D152A) mutant relative to wild-type IspE. For the time course shown in Figure 4A, the concentrations of CDP-ME, CMP and cMEDP are essentially equal after $\sim 12$ minutes. When the concentration of IspE is increased 10 -fold, this equivalency time point drops to $\sim 4$ minutes and is essentially unchanged (Figure $4 \mathrm{~B}$ ). Addition of excess IspE(152A) protein does not substantially alter the time course (Figure 4C). At longer reaction times the "apparent" concentration of CDP-ME levels off while those of the other intermediates decrease. We attribute this to an unidentified product that slowly accumulated during the incubation and migrates between CDP-ME and CMP. A comparison of the overall time courses shown in Figures 4A and 4B reveals that IspE(D152A) does not significantly alter the flux from MEP through CDP-ME and CDP-MEP, consecutively, resulting in cMEDP. The time course experiments with $3.1 \mu \mathrm{M}$ IspE were repeated in assay buffers containing $30 \%(\mathrm{v} / \mathrm{v})$ glycerol in the presence and the absence of the $\operatorname{IspE}(\mathrm{D} 152 \mathrm{~A})$ mutant ${ }^{15}$. The increased viscosity of the medium due to glycerol did not noticeably alter the time course of the reaction (see supporting information). These kinetic results focused on the metabolic flux through a reconstituted pathway of three MEP pathway enzymes strongly argue against enhanced flux due to substrate channeling between the A. tumefaciens IspDF and IspE proteins or to the formation of higher local concentrations of intermediates produced in a multi-enzyme complex.

\section{Discussion}

The bifunctional IspDF enzymes catalyze two nonconsecutive reactions in the MEP pathway, MEP to CDP-ME and CDP-MEP to cMEDP, respectively. The intermediate step, CDP-ME to CDP-MEP, is catalyzed by the ATP-dependent IspE kinase. Sedimentation velocity studies indicate that the IspDF and IspE enzymes from A. tumefaciens and $C$. jejuni associate in solution to form complexes consisting of up to 18 catalytic centers ${ }^{11}$. No evidence was presented for an 18 catalytic center complex formed by association of the IspD, IspE, IspF proteins from $E$. coli ${ }^{11}$. These observations are consistent with a hypothesis that enhanced metabolic flux in the MEP pathway at least from MEP to cMEDP may result by either reducing the distance over which reactive intermediates must diffuse as they move from one active site to another or by providing for direct substrate channeling among the active sites of a preassembled complex of IspDF and IspE ${ }^{11}$.

The formation of multi-protein complexes of biosynthetic enzymes comprising metabolic pathways offers an attractive and powerful mechanism for enhancing the efficiency and flux of moving intermediates through a pathway. Perhaps the most impressive examples of such efficiency and flux are found in polyketide biosynthesis where a combination of multifunctional enzymes and multi-protein complexes permit tethered intermediates to be efficiently shuttled from one active site to the next as an extraordinarily complex set of molecules is assembled $16,17,18$. Several methods have been developed to study substrate channeling between two enzymes 19,20 , including competition experiments that employ a wild type enzyme and a large excess of an inactive mutant that can replace the active enzyme in the multi-enzyme complex. In our experiments, addition of a 10-fold excess of the inactive IspE (D152A) mutant did not alter the time course for three reactions, MEP to CDP-ME to CDPMEP to cMEDP in the middle portion of the MEP pathway of isoprenoid biosynthesis.

The observable flux through a pathway might also increase due to an increase in the local concentration of intermediates when the enzymes are in close proximity 19 . Depending on the structure of the complex, this effect can be enhanced by an increase in the viscosity of the medium 15,21 . Our results demonstrate that increasing the viscosity of the medium by adding 
the microviscogen glycerol does not affect the time course of the consecutive reactions in the presence or absence of IspE(D152A). If the flux from MEP to CDP-ME then to CDP-MEP and finally to cMEDP was enhanced by direct substrate channeling or by an increase in the "local" concentrations of the intermediates due to the close proximity of the actives sites in the complex, incorporation of the inactive mutant should measurably decrease the kinetic efficiency of the biosynthetic steps in question ${ }^{22}$.

Does the fusion of the IspD and IspF genes, in concert with the association of the IspDF and IspE proteins constitute a complex that facilitates the conversion of MEP to cMEDP? Under the conditions tested by our experiments, facilitation of metabolite transfer and production does not occur. Neither of these scenarios was observed.

There are other examples of bifunctional enzymes which do not channel substrates, for example the GlmU protein 23 and phosphoribosyl-anthranilate isomerase-indoleglycerol-phosphate synthase $^{24}$. Even in a case where channeling was "leaky" in a three-reactant system and the proximity of the newly released intermediate increased the probability of it productively reaching the next active site in the pathway, 25 the presence of high concentrations of an inactive mutant for the second step should alter the flux through the three steps. The absence of experimental evidence for an enhancement of metabolic flux through the reactions catalyzed by the IspDF/IspE complex raises the question of what possible utility the IspDF fusion or formation of an IspDF/IspE complex serves in the host cell. At this point, the metabolic logic behind the gene fusion remains unclear and may be related to a yet undiscovered regulatory role in the MEP pathway.

\title{
Supplementary Material
}

Refer to Web version on PubMed Central for supplementary material.

\author{
Acknowledgments \\ This project was supported by NIH Grants GM21328 (CDP) and AI51438 (JPN) and by a postdoctoral fellowship \\ from the Deutsche Forschungsgemeinschaft (FP).
}

\section{REFERENCES}

1. Bochar, DA.; Freisen, JA.; Stauffacher, CV.; Rodwell, VW. Comprehensive Natural Products Chemistry. Cane, D., editor. 2. Pergamon Press; Oxford: 1999. p. 15-44.

2. Rohmer M. The discovery of a mevalonate-independent pathway for isoprenoid biosynthesis in bacteria, algae and higher plants. Nat. Prod. Rep 1999;16:565-574. [PubMed: 10584331]

3. Eisenreich W, Schwarz M, Cartayrade A, Arigoni D, Zenk MH, Bacher A. The deoxyxylulose phosphate pathway of terpenoid biosynthesis in plants and microorganisms. Chem. Biol 1998;5:R221R233. [PubMed: 9751645]

4. Eisenreich W, Rohdich F, Bacher A. Deoxyxylulose phosphate pathway to terpenoids. Trends Plant Sci 2001;6:78-84. [PubMed: 11173292]

5. Rohdich F, Wungsintaweekul J, Fellermeier M, Sagner S, Herz S, Kis K, Eisenreich W, Bacher A, Zenk MH. Cytidine 5'-triphosphate-dependent biosynthesis of isoprenoids: YgbP protein of Escherichia coli catalyzes the formation of 4-diphosphocytidyl-2-C-methylerythritol. Proc. Natl. Acad. Sci. USA 1999;96:11758-11763. [PubMed: 10518523]

6. Kuzuyama T, Takagi M, Kaneda K, Dairi T, Seto H. Formation of 4-(cytidine 5'-diphospho)-2-Cmethyl-d-erythritol from 2- $C$-methyl-d-erythritol 4-phosphate by 2 - $C$-methyl-d-erythritol 4phosphate cytidylyltransferase. Tetrahedron Lett 2000;41:703-706.

7. Luttgen H, Rohdich F, Herz S, Wungsintaweekul J, Hecht S, Schuhr CA, Fellermeier M, Sagner S, Zenk MH, Bacher A, Eisenreich W. Biosynthesis of terpenoids: YchB protein of Escherichia coli phosphorylates the 2-hydroxy group of 4-diphosphocytidyl-2C-methyl-D-erythritol. Proc. Natl. Acad. Sci. USA 2000;97:1062-1067. [PubMed: 10655484] 
8. Herz S, Wungsintaweekul J, Schuhr CA, Hecht S, Lüttgen H, Sagner S, Fellermeier M, Eisenreich W, Zenk MH, Bacher A, Rohdich F. Biosynthesis of terpenoids: YgbB protein converts 4diphosphocytidyl-2- $C$-methyl-d-erythritol 2-phosphate to 2- $C$-methyl-d-erythritol 2,4cyclodiphosphate. Proc. Natl. Acad. Sci. USA 2000;97:2486-2490. [PubMed: 10694574]

9. Testa CA, Lherbet C, Pojer F, Noel JP, Poulter CD. Cloning and expression of IspDF from Mesorhizobium loti. Characterization of a bifunctional protein that catalyzes non-consecutive steps in the methylerythritol pathway. Biochim. Biophys. Acta. In press

10. Gabrielsen M, Rohdich F, Eisenreich W, Grawert T, Hecht S, Bacher A, Hunter WN. Biosynthesis of isoprenoids: a bifunctional IspDF enzyme from Campylobacter jejuni. Eur. J. Biochem 2004;271:3028-3035. [PubMed: 15233799]

11. Gabrielsen M, Bond CS, Hallyburton I, Hecht S, Bacher A, Eisenreich W, Rohdich F, Hunter WN. Hexameric assembly of the bifunctional methylerythritol 2,4-cyclodiphosphate synthase and proteinprotein associations in the deoxy-xylulose-dependent pathway of isoprenoid precursor biosynthesis. J. Biol. Chem 2004;279:52753-52761. [PubMed: 15466439]

12. Bradford MM. A rapid and sensitive method for the quantitation of microgram quantities of protein utilizing the principle of protein-dye binding. Anal. Biochem 1976;72:248-254. [PubMed: 942051]

13. Dam J, Schuck P. Sedimentation Velocity Analysis of Heterogeneous Protein-Protein Interactions: Sedimentation Coefficient Distributions c(s) and Asymptotic Boundary Profiles from Gilbert-Jenkins Theory. Biophys J 2005;89:651-666. [PubMed: 15863474]

14. Miallau L, Alphey MS, Kemp LE, Leonard GA, McSweeney SM, Hecht S, Bacher A, Eisenreich W, Rohdich F, Hunter WN. Biosynthesis of isoprenoids: crystal structure of 4-diphosphocytidyl-2Cmethyl-D-erythritol kinase. Proc. Natl. Acad. Sci. U. S. A 2003;100:9173-9178. [PubMed: 12878729]

15. Otsuki T, Mori M, Tatibana M. Studies on channeling of carbamoyl-phosphate in the multienzyme complex that initiates pyrimidine biosynthesis in rat ascites hepatoma cells. J. Biochem 1982;92:1431-7. [PubMed: 6130083]

16. Beck BJ, Aldrich CC, Fecik RA, Reynolds KA, Sherman DH. Substrate recognition and channeling of monomodules from the pikromycin polyketide synthase. J. Am. Chem. Soc 2003;125:1255112557. [PubMed: 14531700]

17. Hartung IV, Rude MA, Schnarr NA, Hunziker D, Khosla C. Stereochemical assignment of intermediates in the rifamycin biosynthetic pathway by precursor-directed biosynthesis. J. Am. Chem. Soc 2005;127:11202-11203. [PubMed: 16089423]

18. Admiraal SJ, Khosla C, Walsh CT. A Switch for the transfer of substrate between nonribosomal peptide and polyketide modules of the rifamycin synthetase assembly line. J. Am. Chem. Soc 2005;125:13664-13665. [PubMed: 14599196]

19. Geck MK, Kirsch JF. A novel, definitive test for substrate channeling illustrated with the aspartate aminotransferase/malate dehydrogenase system. Biochemistry 1999;38:8032-8037. [PubMed: 10387047] and references cited therein.

20. Anderson KS. Fundamental mechanisms of substrate channeling. Methods Enzymol 1999;308:111145. [PubMed: 10507003]

21. Morgunov I, Srere PA. Interaction between citrate synthase and malate dehydrogenase. Substrate channeling of oxaloacetate. J. Biol. Chem 1998;273:29540-29544. [PubMed: 9792662]

22. Nogaj LA, Beale SI. Physical and kinetic interactions between glutamyl-tRNA reductase and glutamate-1-semialdehyde aminotransferase of Chlamydomonas reinhardtii. J. Biol. Chem 2005;280:24301-24307. [PubMed: 15890644]

23. Gehring AM, Lees WJ, Mindiola DJ, Walsh CT, Brown ED. Acetyltransfer precedes uridylyltransfer in the formation of UDP-N-acetylglucosamine in separable active sites of the bifunctional GlmU protein of Escherichia coli. Biochemistry 1996;35:579-585. [PubMed: 8555230]

24. Eberhard M, Tsai-Pflugfelder M, Bolewska K, Hommel U, Kirschner K. Indoleglycerol phosphate synthase-phosphoribosyl anthranilate isomerase: comparison of the bifunctional enzyme from Escherichia coli with engineered monofunctional domains. Biochemistry 1995;34:5419-5428. [PubMed: 7727400]

25. Kis K, Bacher A. Substrate channeling in the lumazine synthase/riboflavin synthase complex of Bacillus subtilis. J. Biol. Chem 1995;270:16788-16795. [PubMed: 7622491] 


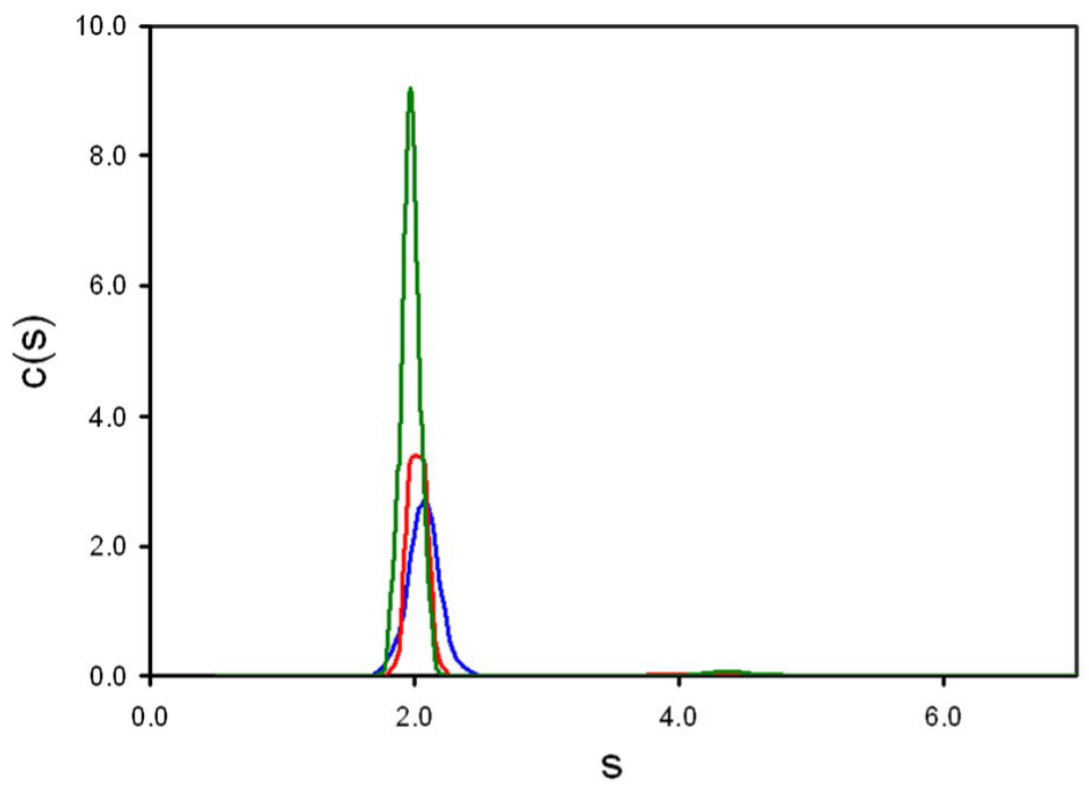

Figure 1.

Sedimentation velocity experiments performed with $25 \mu \mathrm{M}$ wild-type IspE (blue), $28 \mu \mathrm{M}$ IspE (D152A) (red), and $50 \mu \mathrm{M}$ IspE(D152A) (green). 


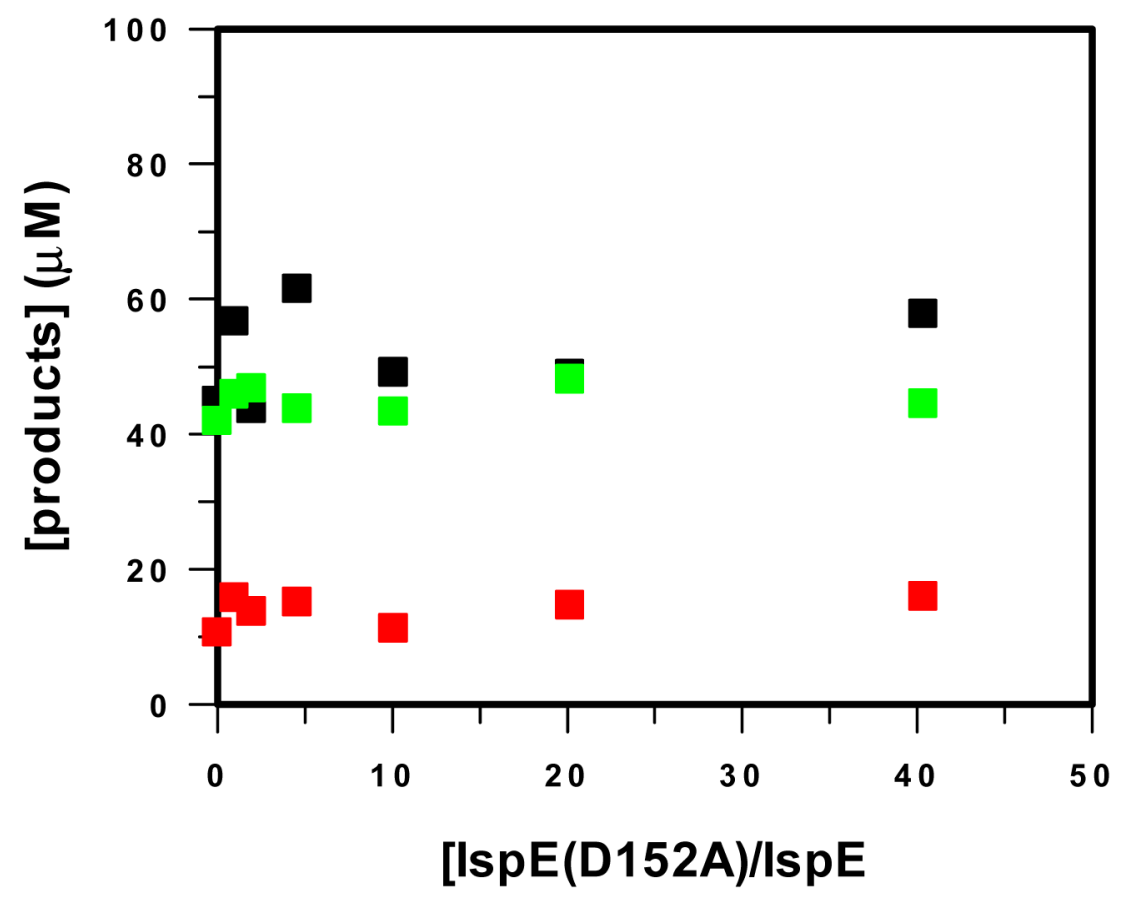

Figure 2.

Product distributions for A. tumefaciens IspDF and IspE with added IspE(D152A). CDP-ME $(\varpi)$, CDP-MEP ( $)$, CMP or cMEDP ( $)$ ). Substrate and enzyme concentrations were as follows: $150 \mu \mathrm{M} \mathrm{CTP}(40 \mu \mathrm{Ci} / \mu \mathrm{mol}), 150 \mu \mathrm{M}$ ATP $(320 \mu \mathrm{Ci} / \mu \mathrm{mol}), 500 \mu \mathrm{M} \mathrm{MEP}$ (racemic), $0.24 \mu \mathrm{M}$ IspDF, $0.31 \mu \mathrm{M}$ IspE and different concentrations $(0,0.3,0.6,1.4,3.1,6.3,12.5 \mu \mathrm{M})$ of IspE (D152A) in a final volume of $50 \mu \mathrm{L}$. 


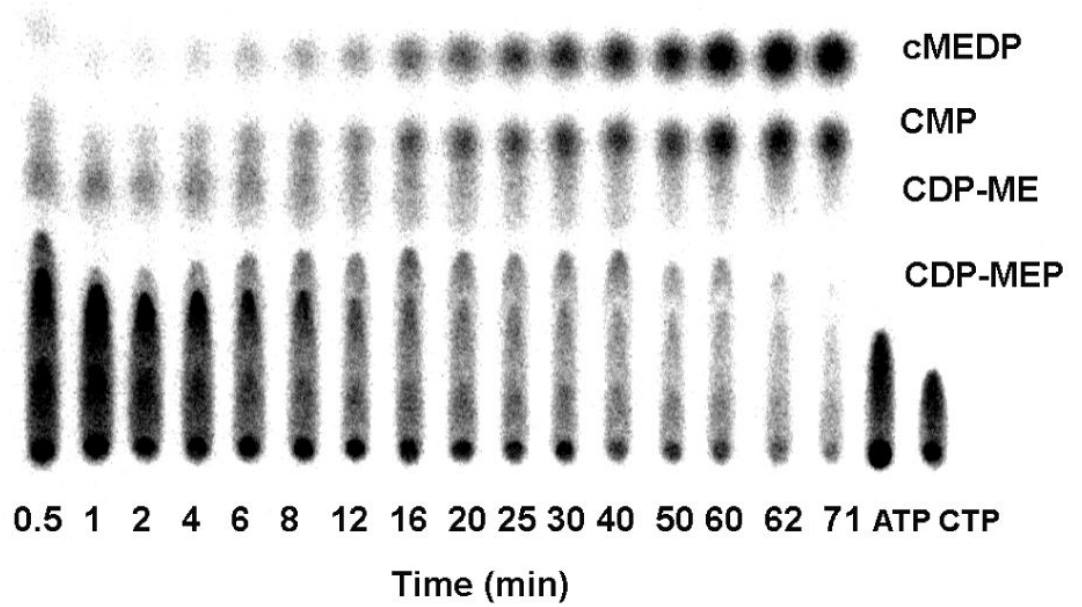

Figure 3.

A TLC plate showing the time course for the formation of CDP-ME, CDP-MEP, CMP, cMEDP catalyzed by IspDF $(0.24 \mu \mathrm{M})$, IspE $(0.31 \mu \mathrm{M})$. At $61 \mathrm{~min}$ an additional $0.5 \mu \mathrm{g}$ portion of each enzyme were added. ATP and CTP lanes represent blank controls without enzyme. 


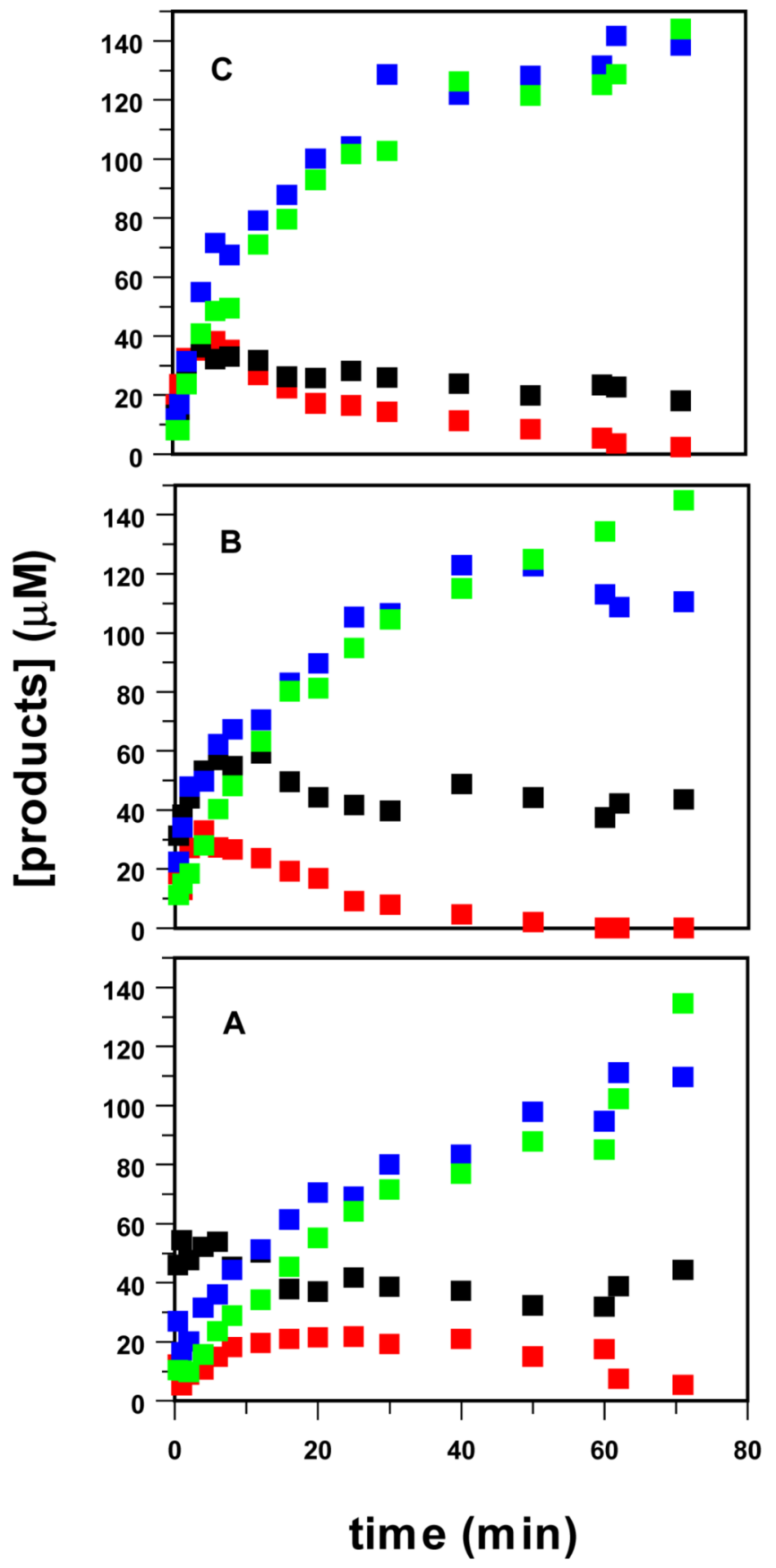

Figure 4.

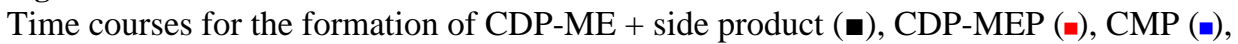
cMEDP ( () . Panel A: IspDF $(0.24 \mu \mathrm{M})$ and IspE $(0.31 \mu \mathrm{M})$. Panel B: IspDF $(0.24 \mu \mathrm{M})$ and IspE $(3.1 \mu \mathrm{M})$. Panel C: IspDF $(0.24 \mu \mathrm{M})$, IspE $(3.1 \mu \mathrm{M})$, and IspE(D152A) $(31.2 \mu \mathrm{M})$. 


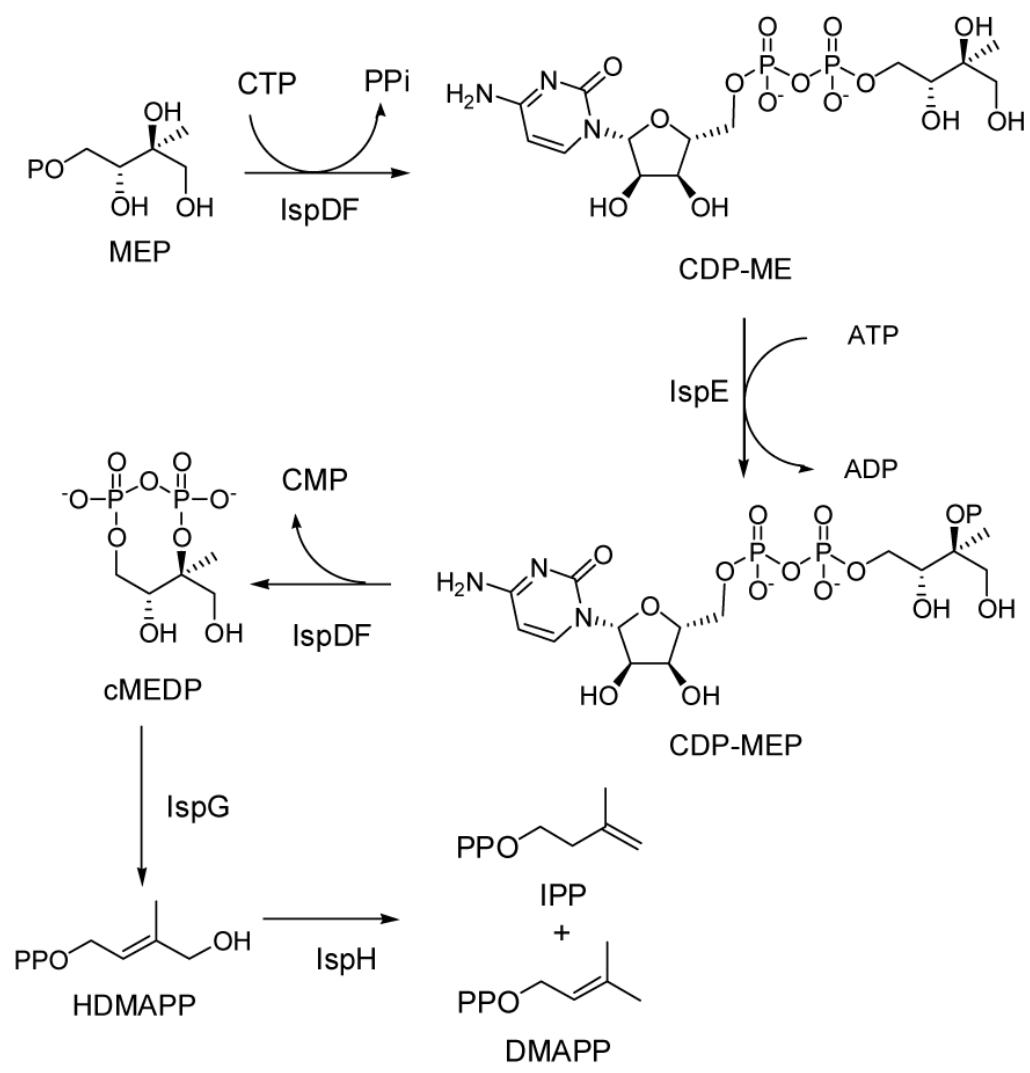

Scheme 1.

The MEP pathway for biosynthesis of isoprenoid compounds. 
Table 1

Oligonucleotides used in this study

\begin{tabular}{cll}
\hline Enzyme & Oligonucleotide & Sequence \\
& & \\
\hline IspDF & CL0610DFC58sen & 5'AAAGCCCATATGCAGGAAAGTACTATGAAATTCGGCATCGTCATC 3' \\
IspE & CL0610DFC58ant & 5'AAAGCCGGATCCATTATAGCGGTCTGCCTTGGTAGACGACGGTG 3' \\
& CL0429EC58sen & 5'AAAAGCCATATGCGCCTGCATGAGGTTCCGGGGC 3' \\
IspE(D152A) & CL0429EC58ant & 5'AAAGCCGGATCCATTAAATGGTCCGCGTTGCGTGAAAATACCAGC 3' \\
& AMAISPE007 & 5' GAAACTGGGCGCGGCTGTACCGATGTGC 3' \\
& GC-AMAISPE007 & 5' GCACATCGGTACAGCCGCGCCCAGTTC 3' \\
\hline
\end{tabular}

